\title{
Genome characterization of Pseudomonas phages BIM BV-45 and BIM BV-46 - the components of biopesticide Multiphage to control bacterial diseases of vegetable crops \\ Pilipchuk T.A., Valentovich L.N., Kalamiyets E.I.
}

Institute of Microbiology, NAS of Belarus, Minsk, Republic of Belarus

E-mail: tanya.pilipchuk@tut.by

Key message. The conducted investigation resulted in full nucleotide sequencing of Pseudomonas phage BIM BV-45 and Pseudomonas phage BIM BV-46 (registration numbers in GenBank NCBI MT094430 and MT094431) - components of biopesticide Multiphage

Keywords: genome, bacteriophages, Pseudomonas

Nowadays due to the growing challenge of antibiotic resistance in pathogenic bacteria application of lytic bacteriophages appears to be an alternative strategy to combat them. It seems natural therefore that search and characterization of bacteriophages will lay the basis for their target-oriented use as biological control agents. Biopesticide Multiphage developed at Institute of Microbiology, NAS of Belarus incorporates six phages protecting vegetable cultures from phytopathogenic pseudomonades.

Aim of the study was analysis of nucleotide sequences of genomes of two bacteriophages Pseudomonas phage BIM BV-45 and Pseudomonas phage BIM BV-46 from biopesticide Multiphage.

Nucleotide sequencing was carried out on MiSeq platform (Illumina). Automatic annotation of genetic fragments was performed using Prokka software complemented with databases InterProScan, CDSearch, BLASTp. Phage promoters were detected with the aid of PHIRE. Nucleotide sequences of phages BIM BV-45 and BIM BV-46 are deposited in GenBank NCBI library under registration numbers MT094430 and MT094431, respectively.

Analysis of full nucleotide sequence of phage BIM BV-45 sized 40383 bp (58\% GC content) led us to deduce that phage genome is represented by linear double-stranded DNA identical by $97 \%$ to Pseudomonas phage Andromeda (KX458241) belonging to genus Befseprivirus, family Podoviridae, order Caudovirales. 46 open reading frames probably determining stages of the lytic cycle and 4 sigma-70 promoters were revealed within phage genome sequence.

Genome of bacteriophage BIM BV-46 also comprised linear double-stranded DNA consisting of $38860 \mathrm{bp}$ (56\% GC content). The closest similarity (97.8\%) was shown to Pseudomonas phage Pf-10 (KP025626), belonging to T7 phages of genus Autographivirinae, family Podoviridae, order Caudovirales. 38 open reading frames, 4 sigma-70 promoters and 9 specific phage promoters were found in BIM BV-46 genome sequence.

The amino acid residue sequences of the BIM BV-45 phage were 95-99\% identical to Pseudomonas phage Andromeda isolated against Pseudomonas syringae bacteria. The second similar to phage BIM BV-45 was Pseudomonas phage Bf7 (JN991020) inhibiting the growth of bacteria Pseudomonas tolaasii, the causative agent of brown spotting of champignons. The amino acid sequences of Pseudomonas phage Bf7 were similar to 75-96\% of all investigated proteins of phage BIM BV45 except for DNA ligase, which was $96 \%$ identical to the sequence of Pseudomonas phage Andromeda. The sequences of aminoacid residues of DNA endonuclease from phage BIM BV-45 were different from the proteins of the above-mentioned phages but showed small similarity (63\%) to Pseudomonas phage PollyC (MG775261), causing lysis of the sweet cherry pathogen Pseudomonas syringae.

Most sequences of amino acid residues of phage BIM BV-46 were similar by 97-100\% to proteins of Pseudomonas phage Pf10 (KP025626), which is also one of the components of biopesticide Multiphage (BIM BV-61) and is active against phytopathogenic bacteria Pseudomonas fluorescens, Pseudomonas syringae and Pseudomonas putida. In the bacteriophage BIM BV-46, the proteins responsible for the synthesis of endonuclease and endolysin were 99-100\% identical to the Pseudomonas phage Phi-S1 (JX173487) proteins, while the amino acid sequences of the tail, the internal proteins of the virion and holine of BIM BV-46 showed identity 96-98\%. The phage Pseudomonas phage Phi-S1 was isolated against the bacterium Pseudomonas fluorescens in a wastewater treatment plant and lysed most of the fluorescent species Pseudomonas and one strain of Pseudomonas stutzeri.

Summing up, based on genome structure, phages Pseudomonas phage BIM BV-45 and Pseudomonas phage BIM BV-46 constituting active principle of biopesticide of Multiphage are viruses referred to order Caudovirales, family Podoviridae, genera Bifseprivirus (BIM BV-45) and Autographivirinae (BIM BV-46). Genetic correlations of the studied phage genomes with nucleotide sequences of other Pseudomonas phages (deposited in GenBank NCBI) and small percentage of identity in the examined amino acid sequences indicate mosaic structure of the phages and mutational shifts arising in the course of phage genome evolution. The genetic diversity of bacteriophages BIM BV-45 and BIM BV-46 evidences a potentially broad spectrum of lytic activity of biopesticide of Multiphage toward phytopathogenic bacteria of genus Pseudomonas.

This work was financially supported by the Belarusian republican foundation for fundamental research (grant № 520P-078). 